\title{
Using Mobile Health Technology and Social Media for the Prevention and Early Detection of Skin Cancer
}

\author{
Monika Janda ${ }^{a}$ H. Peter Soyer ${ }^{b, c}$ \\ ${ }^{a}$ Centre for Health Services Research, Faculty of Medicine, University of Queensland, Brisbane, QLD, Australia; ${ }^{\mathrm{b}}$ The \\ University of Queensland Diamantina Institute, Dermatology Research Centre, University of Queensland, Brisbane, \\ QLD, Australia; 'Dermatology Department, Princess Alexandra Hospital, Brisbane, QLD, Australia
}

Skin cancer, including melanoma, keratinocyte and other rare skin cancers, represent the most commonly diagnosed forms of cancer in Caucasian populations worldwide [1]. In 2017, there were 309,000 new cases of melanoma (62,000 deaths) as well as 7.7 million cases of keratinocyte skin cancers (65,000 deaths) [2]. In Australia, excluding keratinocyte cancers, melanoma is the fourth most commonly diagnosed invasive cancer and the most common cancer diagnosed in young people aged 15-39 years $[3,4]$. Similarly in the USA, melanoma is a major public health problem as one of the most rapidly increasing cancers [5]. Melanoma and other skin cancers are primarily caused by overexposure to ultraviolet radiation [6]. Improvements in both primary prevention and early detection efforts will be necessary to reduce skin cancer incidence and mortality rates.

Australian adults are still being sunburnt on a regular basis, despite the known high risks of skin cancer. Up to $17 \%$ of adults reported being sunburnt on an average summer weekend in the most recent Australian nationwide survey conducted in 2016-2017 [7]. This high event rate also finds reflectance in social media, which is now a ubiquitous form of communication especially amongst young people [8]. In this issue of Dermatology, Stekelen-

karger@karger.com

(C) 2019 S. Karger AG, Basel

www.karger.com/drm

Karger burg et al. [9] report a content analysis of tweets mentioning sunburns, extracting major themes of how Australian Twitter users report about their experiences. The study found that many Twitter users depict their sunburns in a positive, affective and humorous way, which adds to the growing concerns that Australians are still complacent about sunburn. A notable theme was "Australian identity" where sunburns were described as a normal part of the outdoors lifestyle in Australia. This study indicates that social media need to be used increasingly to influence how sunburn is perceived, reduce its depiction as a "normal" part of living in a sunny country and encourage better sun protection and skin cancer risk-reducing behaviours.

The paper by Silva et al. [10] revealed that the daily number of tweets is greatly affected by environmental conditions such as high ambient temperature. The study analysed Twitter posts in Australia longitudinally over the summer of 2018/2019 to establish skin cancer and sun-related communication trends. Results demonstrate that Australians are using Twitter to communicate about

This article is part of the Nevi Article Series 
their sun-related and skin cancer experiences and to share advice on this matter, however tweet less frequently about ongoing skin cancer-related public health campaigns. In a significant change from how health communication was traditionally distributed from health authorities to the public, today celebrities, non-health-related organisations with a high number of followers, and individuals sharing their own skin cancer experiences were most influential when tweeting about skin cancer prevention.

Mobile health (mHealth) is characterised by the rapidly expanding use of mobile technologies in health care. There is a vast potential for mHealth interventions to facilitate consumer adoption of healthy lifestyle choices and delivery of prompt health services. Incorporating mobile teledermoscopy into both the direct-to-consumer and doctor-to-doctor settings may facilitate the earlier detection of melanoma. In the direct-to-consumer setting, consumers (traditionally called patients) can utilise their smartphone with or without their own dermatoscope attachment and a corresponding mHealth application (app) for convenient use at home. This setting includes lesions self-selected by patients for review or identified by doctors for monitoring between clinical visits. Both settings move towards self-tracking of suspicious skin lesions in the spirit of the "Quantified Self" website and movement in which individuals become more involved in the management of their health [11]. In the doctor-to-doctor setting, images are triaged or sent for second opinion between health professionals with the potential to replace the in-person visits. Kong et al. [12] interviewed 28 people about their thoughts on mobile teledermoscopy and reported their findings in this issue. Convenience, access and cost were the main contributing factors for participants' potential use of direct-to-consumer and doctor-to-doctor mobile teledermoscopy settings.
Further, in this issue of Dermatology Horsham et al. [13] and Janda et al. [14] explore consumer acceptance of mobile teledermoscopy for the early detection of skin cancer following involvement in a clinical trial with hands-on experience with the use of mobile teledermoscopy. Participants in this study $(n=98)$ used mobile teledermoscopy according to the direct-to-consumer setting. Participants completed an online survey on mobile teledermoscopy acceptance based on the technology acceptance model both before and after use. This study found that participant's acceptance of mobile teledermoscopy decreased after use. This is the first study to assess acceptance before and after the practical experience with mobile teledermoscopy.

In summary, as the landscape of mHealth technology continues to expand in its scope and importance, research on how these technologies can best be integrated into consumers' everyday lives is required, and the papers in this issue provide details on the various aspects of this ubiquitous trend.

\section{Disclosure Statement}

H.P.S. is a shareholder of MoleMap NZ Ltd. and e-derm consult $\mathrm{GmbH}$, and undertakes regular teledermatological reporting for both companies. H.P.S. is a Medical Consultant for Canfield Scientific Inc. and MetOptima, and a Medical Advisor for First Derm.

\section{Funding Sources}

This research is funded by a research grant awarded to M.J. from the National Health and Medical Research Council APP1113962 and APP1099021. M.J. is funded by a TRIP Fellowship APP1151021. H.P.S. is also funded by the Medical Research Future Fund - Next Generation Clinical Researcher's Program Practitioner Fellowship APP1137127.

\section{References}

1 Apalla Z, Lallas A, Sotiriou E, Lazaridou E, Ioannides D. Epidemiological trends in skin cancer. Dermatol Pract Concept. 2017 Apr; $7(2): 1-6$.

2 Fitzmaurice C, Abate D, Abbasi N, Abbastabar H, Abd-Allah F, Abdel-Rahman O, et al.; Global Burden of Disease Cancer Collaboration. Global, Regional, and National Cancer Incidence, Mortality, Years of Life Lost, Years Lived With Disability, and Disability-Adjusted Life-Years for 29 Cancer Groups, 1990 to 2017: A Systematic Analysis for the Global Burden of Disease Study. JAMA Oncol. 2019 Sep.

3 Australian Institute of Health and Welfare. Cancer in Australia. Canberra: AIHW; 2019 [cited 2019 Aug 12]. Cancer series No. 119. Available from: https://www.aihw.gov.au/ getmedia/8c9fcf52-0055-41a0-96d9-f81b0feb $98 \mathrm{cf} /$ aihw-can-123.pdf.aspx?inline $=$ true

4 Australian Institute of Health and Welfare. Cancer in adolescents and young adults in Australia. Canberra: AIHW; 2018 [cited 2019 Aug 12]. Cat. No. 110. Available from: https:// www.aihw.gov.au/getmedia/ed22109b-ab234273-8d23-7949a8922ea2/aihw-can-110.pdf. aspx?inline $=$ true

5 Feigelson HS, Powers JD, Kumar M, Carroll NM, Pathy A, Ritzwoller DP. Melanoma incidence, recurrence, and mortality in an integrated healthcare system: A retrospective cohort study. Cancer Med. 2019 Aug;8(9):450816.

6 Narayanan DL, Saladi RN, Fox JL. Ultraviolet radiation and skin cancer. Int J Dermatol. 2010 Sep;49(9):978-86. 
7 Cancer Council Australia. The National sun Protection Survey [Available from: https:// www.cancersa.org.au/stay-up-to-date/pressreleases/details/we-re-still-a-sunburnt-country.

8 Moreno MA. Social networking sites and adolescents. Pediatr Ann. 2010 Sep;39(9):565-8.

9 Stekelenburg N, Horsham C, O'Hara M, Janda M. Using social media to determine the affective and cognitive components of tweets about sunburn. Dermatology. 2019. DOI: 506102.
10 Silva CV, Jayasinghe D, Janda M. What can Twitter tell us about the public's skin cancer prevention knowledge, attitudes and intentions? Dermatology. 2019. DOI: 506458.

11 Quantified Self [cited 2019 September 11]. Available from: http://quantifiedself.com/.

12 Kong F, Horsham C, Rayner R, Simunovic M, Soyer HP, Janda M. Consumer preferences for skin cancer screening using mobile teledermoscopy: a qualitative study. Dermatology. 2019. DOI: 505620.
13 Horsham C, Snoswell C, Vagenas D, Loescher LJ, Gillespie N, Soyer HP, et al. Is teledermoscopy ready to replace face to face examinations for the early detection of skin cancer? Consumer views, technology acceptance and satisfaction with care. Dermatology. 2019. DOI: 506154

14 Janda M, Horsham C, Koh U, Gillespie N, Loescher LJ, Vagenas D, et al. Redesigning Skin Cancer Early Detection and Care Using a New Mobile Health Application: Protocol of the SKIN Research Project, a Randomised Controlled Trial. Dermatology. 2019;235(1): 11-8. 